Original Article Journal of Epilepsy Research pISSN 2233-6249 / eISSN 2233-6257

Received September 2, 2017

Accepted November 27, 2017

Corresponding author: Yun-Jin Lee

Department of Pediatrics, Pusan National

University Children's Hospital, Pusan

National University School of Medicine,

20 Geumo-ro, Meulgeum-eup, Yangsan

50612, Korea

Tel. $+82-55-360-2180$

Fax. $+82-55-360-2181$

E-mail; jinnyeye@hanmail.net

\section{Longitudinal Change in Thyroid Hormone Levels in Children with Epilepsy on a Ketogenic Diet: Prevalence and Risk Factors}

\author{
Yun-Jin Lee ${ }^{1,2}$, Sang Ook Nam ${ }^{1,2}$, Kyung-Min Kim³ ${ }^{3}$ Young Mi Kim ${ }^{4}$, Gyu Min Yeon ${ }^{5}$ \\ ${ }^{1}$ Department of Pediatrics, Pusan National University Children's Hospital, Pusan National University School of \\ Medicine, Yangsan; ${ }^{2}$ Research Institute for Convergence of Biomedical Science and Technology, Pusan National \\ University Yangsan Hospital, Yangsan; ${ }^{3}$ Department of Pediatrics, Good Gang-An Hospital, Busan; ${ }^{4}$ Department of \\ Pediatrics, Pusan National University Hospital, Busan; ${ }^{5}$ Department of Pediatrics, Kosin University Gospel \\ Hospital, Kosin University, Busan, Korea
}

\begin{abstract}
Background and Purpose: The aim of this study is to evaluate the prevalence of hypothyroidism and the change of thyroid hormone level in the children with epilepsy on a ketogenic diet (KD).

Methods: The levels of serum free thyroxine (fT4) and thyroid-stimulation hormone (TSH) were measured at the start of the KD and at 6- to 12-month intervals in children with intractable epilepsy. Hypothyroidism was defined as fT4 level $<0.8 \mathrm{ng} / \mathrm{dL}$ and TSH level $>6.0 \mu \mathrm{lU} / \mathrm{mL}$.

Results: A total of 28 children (17 boys and 11 girls) were enrolled in the study. The mean age of onset of seizure was $1.4 \pm 1.6$ years, the mean age of the start of the KD was $3.2 \pm 2.4$ years, and the mean duration of KD was $1.9 \pm 1.5$ years. Overall, there was no significant longitudinal change in the mean fT4 $(0.99 \pm 0.25$ vs. $0.94 \pm 0.71 \mathrm{ng} / \mathrm{dL}, p=0.28)$ and TSH $(2.94 \pm 1.32 \mathrm{vs} .3 .18 \pm 1.21 \mu \mathrm{lU} / \mathrm{mL}, p=0.44)$ levels from the start of the KD to last follow-up. The patients with a younger age of seizure onset, earlier initiation of KD, and higher serum levels of cholesterol and triglyceride had a significant decrease in fT4 levels and increase in TSH levels during the $K D$. Sex, duration of the seizure or KD therapy, seizure types, seizure frequency, seizure outcomes, brain lesion, ratio of $\mathrm{KD}$, and being overweight did not affect the longitudinal change of fT4 and TSH levels during KD.
\end{abstract}

Conclusion: Thyroid function had no significant longitudinal decrease in pediatric epilepsy during KD therapy. However, careful monitoring of the serum levels of fT4/TSH should be recommended in children on KDs, especially in those with earlier seizure onset, earlier start of KD, and higher levels of lipid profiles. (2017;7:99-105)

Key words: Thyroid hormones, Hypothyroidism, Epilepsy, Child, Ketogenic diet

\section{Introduction}

Antiepileptic drugs (AEDs) are used to treat epilepsy and other conditions including psychiatric disorders, neuropathic pain, and migraine. The prevalence of AED use varies between countries, ranging from $0.97 \%$ to $1.38 \%$ of the entire population. ${ }^{1}$ Previous studies advise that AEDs might unfavorably influence endocrine homeostasis (e.g., thyroid hormones). Hypothyroidism is of particular concern because it could lead to diastolic hypertension, dyslipidemia, coagulopathy, and atherosclerosis, which in turn could increase the risk of a cardiovascular event and mortality. ${ }^{2}$ Thyroid hormones also play an important role in protein synthesis in tissues; thus, disturbances of thyroid function may hinder growth and development in children. ${ }^{3}$

It is well-known that administration of AEDs may disturb thyroid function in adults. ${ }^{4}$ Different thyroid dysfunctions and subclinical hypothyroidism have been reported in adults. ${ }^{5}$ However, there are few studies in children regarding the effects of AEDs on thyroid functions. ${ }^{6}$ Carbamazepine and valproic acid are the most commonly used AEDs in children with partial or generalized epilepsy. Although they are well tolerated, they have subtle effects on thyroid function. ${ }^{6}$ The results of the few studies investigating thyroid function in pediatric epilepsy with AEDs showed deleterious effects on thyroid func- 
tion to varying degrees during a 12-month therapy period. ${ }^{7-9}$

The ketogenic diet (KD) is an established treatment for medically refractory epilepsy dating back to the 1920s, with documented broad-spectrum efficacy, ${ }^{10}$ and is most often used in children. Dietary treatment has potential adverse effects; most commonly gastrointestinal side effects, weight loss, and hyperlipidemia. Other potential side effects can result from vitamin and mineral deficiencies secondary to restricting carbohydrates (e.g., osteopenia, osteoporosis, and cardiomyopathy). ${ }^{11}$ However, there are no studies, to the best of our knowledge, that evaluate the longitudinal change of the levels of serum free thyroxine (fT4) and thyroid-stimulating hormone (TSH) among children with epilepsy on a KD.

Therefore, the aim of this study is 1) to examine the longitudinal change of the serum fT4 and TSH levels in children with epilepsy on $\mathrm{KD}$ therapy (> 6 months) and 2) to evaluate the potential risk factors for negative change of thyroid function during the KD in a tertiary pediatric epilepsy clinic.

\section{Methods}

\section{Patients}

A retrospective longitudinal cohort study of children with medically intractable epilepsy on KD was undertaken between January 2012 and May 2016, at a pediatric neurology clinic affiliated with Pusan National University Children's Hospital in South Korea. The inclusion criteria of the study subjects were defined as follows: 1) the range of patients' age was between 6 months and 18 years, 2) patients were followed-up for at least 6 months after the start of the $K D, 3$ ) their serum $\mathrm{FT} 4$ and TSH levels were measured before the start of $\mathrm{KD}$ treatment and at 6 - to 12-month intervals thereafter, and 4) no AEDs were changed during the KD. The exclusion criteria were as follows: 1) presence of progressive neurological, thyroid, or other metabolic diseases, and other chronic diseases (e.g., cancer, gastrointestinal disorders, liver insufficiency, diabetes mellitus, chromosomal disease), 2) intake of any drug within the past 6 months that could affect thyroid function, and 3) those with a family history of hypothyroidism or endocrine dysfunction.

\section{Methods}

The levels of serum $\mathrm{fT} 4$ and TSH of all patients were measured before the start of the KD (fT4-1, TSH-1; baseline-fT4 and TSH) and at 6- to 12-month intervals thereafter (fT4-2, TSH-2; fT4 and TSH measured at the last follow-up visit). Blood samples were taken between 8
AM and $10 \mathrm{AM}$ after an overnight fast, and measurement of $\mathrm{fT} 4$ and TSH levels were performed on the same day. All hormonal analyses were performed by chemiluminescent immunoassay (ADVIA Centaur XP device, Siemens Healthcare Firm, USA). Reference ranges for the serum levels were 0.8 to $2.2 \mathrm{ng} / \mathrm{dL}$ for fT4, and 0.7 to $5.5 \mu \mathrm{lU} / \mathrm{mL}$ for TSH. The serum level of fT4 was considered low when it was lower than $0.8 \mathrm{ng} / \mathrm{dL}$ (low-fT4), and the serum TSH level was considered high when it was higher than $6.0 \mu \mathrm{lU} / \mathrm{mL}$ (high-TSH) in our study. The difference or change between the serum levels at baseline and the last follow-up test was investigated as the fT4-D (the value of fT4-2 minus fT4-1) and TSH-D (the value of TSH-2 minus TSH-1).

Detailed information was evaluated by comprehensively reviewing the patients' medical records, which included sex, age of seizure onset ( $<1$ vs. $\geq 1$ year of age) and KD initiation ( $<2$ vs. $\geq 2$ years of age), seizure type, duration of seizure ( $<3$ vs. $\geq 3$ years), AEDs medication ( $<3$ vs. $\geq 3$ years), and $K D$ ( $<2$ vs. $\geq 2$ years), underlying etiologies, seizure outcomes $(\geq 50 \%$ vs. $<50 \%$ reduction of seizure frequency), ratio of $K D(\leq 3: 1$ vs. $4: 1)$, urine ketone $(\leq 3+$ vs. $4+$ ), body mass index (BMI, $<85 \%$ vs. $\geq 85 \%$ ), and serum levels of lipid profiles (total cholesterol, $>200 \mathrm{vs}$. $\leq 200 \mathrm{mg} / \mathrm{dL}$; triglyceride, $>300 \mathrm{vs}$. $\leq 300 \mathrm{mg} / \mathrm{dL}$ ). Patients with AEDs were classified as taking AEDs that have been known as drugs 'related to the thyroid function (AEDs-A)' or 'not related to the thyroid function (AEDs-B).' AEDs-A included valproate, carbamazepine, oxcarbazepine, or phenobarbital. Etiologies were defined as magnetic resonance imaging (MRI)-negative if no known etiology had been identified and the brain MRI was normal. Etiologies were defined as MRI-positive if there was a brain lesion present, such as from previously acquired brain injuries or developmental brain malformations. A BMI of greater than the 85th percentile for their age was defined as being overweight.

\section{Standard protocol approvals, registrations, and patient consent}

Ethical permission for this study was granted by the Institutional Review Board of Pusan National University Yangsan Hospital (number: 05-2017-066), and fully informed written consent was obtained from each participant.

\section{Statistical analysis}

Statistical analyses were performed with SPSS 15.0 software (SPSS, Inc., Chicago, IL, USA) using raw scores. For the initial/last-fT4 and TSH (fT4-1 vs. fT4-2; TSH-1 vs. TSH-2) comparison in all patients, a paired $t$-test was used to evaluate the significant changes between 
the corresponding values (fT4-D, TSH-D). Two-tailed Chi-square and Student's $t$-tests were used to evaluate the significant differences in dependent, categorical, and continuous variables between groups. Multivariate logistic regression was used to identify the variables that were independently associated with a longitudinally significant decrease or increase in the fT4 and TSH levels. Sex, age, duration of seizure, AEDs medication and KD, underlying etiologies, seizure outcomes, ratio of KD, BMI, and serum levels of lipid profiles were all entered as independent variables. Statistical significance was determined as a 2-tailed $p$ value of $<0.05$.

\section{Results}

\section{Change in the serum levels of fT4 and TSH from baseline to last follow-up in total patients}

A total of 28 children were included in this study. The patients' age ranged from 6 months to 9.9 years, with a mean age of 3.2 years ( \pm 2.4 years). Seventeen $(60.7 \%$ ) were boys. The mean duration of KD was $1.9 \pm 1.5$ years. The demographic profiles of the total patients are presented in Table 1. Before the start of KD for all patients, the mean values of fT4- 1 and TSH- 1 levels at baseline were $0.99 \pm 0.25$ $\mathrm{ng} / \mathrm{dL}$ and $2.94 \pm 1.32 \mu \mathrm{lU} / \mathrm{mL}$. The mean values of fT4-2 and TSH-2 levels at the last follow-up were $0.94 \pm 0.71 \mathrm{ng} / \mathrm{dL}$ and $3.18 \pm 1.21$ $\mu \mathrm{UU} / \mathrm{mL}$, which showed no longitudinally significant change (fT4-D $=$ $-0.05 \pm 0.18 \mathrm{ng} / \mathrm{dL}, p=0.28 ; \mathrm{TSH}-\mathrm{D}=0.24 \pm 1.35 \mu \mathrm{lU} / \mathrm{mL}, p=$

Table 1. Demographic profiles of the patients

\begin{tabular}{lc}
\hline & Value \\
\hline Total & $28(100.0)$ \\
Male & $17(60.7)$ \\
Age at seizure onset (years) & $1.4 \pm 1.6(0.1-5.3)$ \\
Duration of seizure before KD (years) & $3.7 \pm 2.8(0.3-9.9)$ \\
Age at start of KD (years) & $3.2 \pm 2.4(0.5-9.9)$ \\
Duration of KD (years) & $1.9 \pm 1.5(0.5-5.4)$ \\
Seizure type & $16(57.1)$ \\
$\quad$ Single type & $12(42.9)$ \\
$\quad$ Multiple type $(\geq 2)$ & $9(32.1)$ \\
Frequency of seizure & $19(67.9)$ \\
$\quad<50 \%$ per week & $20(71.4)$ \\
$\quad$ Nearly daily seizure & $13(46.4)$ \\
MRI lesion & $15(53.6)$ \\
Ratio of KD & \\
$4: 1 / 3: 1 / 2: 1 / 1: 1$ & $16(57.1)$ \\
$\leq 3: 1$ & $12(42.9)$ \\
Urine ketone & \\
$4+$ & \\
$\leq 3+$ &
\end{tabular}

Values are presented as number (\%) unless otherwise indicated. $\mathrm{KD}$, ketogenic diet; $\mathrm{MRI}$, magnetic resonance imaging.
0.44) (Fig. 1).

\section{Factors for aggravation of decreased thyroid function in pediatric epilepsy on KD}

We investigated the potential risk factors affecting the change of fT4 and TSH levels from the baseline to the last follow-up during KD therapy (Table 2). Children of a younger age at seizure onset ( $<1 \mathrm{vs.}$ $\geq 1$ year of age) had a greater negative effect for the change of fT4-D $(-0.11 \pm 0.21$ vs. $0.03 \pm 0.07 \mathrm{ng} / \mathrm{dL}, p=0.02)$ and TSH-D (0.72 \pm 1.48 vs. $-0.39 \pm 0.83 \mu \mathrm{lU} / \mathrm{mL}, p=0.04$ ) levels than those of an older age at seizure onset. Patients of a younger age at initiation of the KD ( $<2$ vs. $\geq 2$ years of age) had a greater negative effect for the change of fT4-D (-0.18 \pm 0.18 vs. $0.04 \pm 0.09 \mathrm{ng} / \mathrm{dL}, p<0.01)$ and TSH-D $(1.33 \pm 1.07$ vs. $-0.57 \pm 0.88 \mu \mathrm{lU} / \mathrm{mL}, p<0.01)$ levels than those of an older age at initiation of the KD. Those with higher serum levels of total cholesterol (> $200 \mathrm{vs}$. $\leq 200 \mathrm{mg} / \mathrm{dL}$ ) and triglyceride (> 300 vs. $\leq 300 \mathrm{mg} / \mathrm{dL}$ ) showed a significant effect for the change of fT4-D $(-0.11 \pm 0.22$ vs. $0.18 \pm 0.08 \mathrm{ng} / \mathrm{dL}, p=0.04 ;-0.14 \pm 0.18$ vs. $0.04 \pm 0.11 \mathrm{ng} / \mathrm{dL}, p<0.01)$ and TSH-D $(0.98 \pm 1.29$ vs. $-0.60 \pm$ $0.83 \mu \mathrm{UU} / \mathrm{mL}, p<0.01 ; 1.12 \pm 1.27$ vs. $-0.63 \pm 0.72 \mu \mathrm{UU} / \mathrm{mL}, p<$ 0.01 ) as compared to those with lower levels of total cholesterol or triglyceride.

Sex, duration of the seizure, AEDs or KD therapy, seizure type, seizure frequency, outcome of seizure reduction, type of AEDs, brain MRI lesion, ratio of $\mathrm{KD}$, levels of urine ketone, and being overweight (BMl of $\geq 85$ th percentile) did not affect the longitudinal change of fT4 and TSH from baseline to last follow-up during KD.
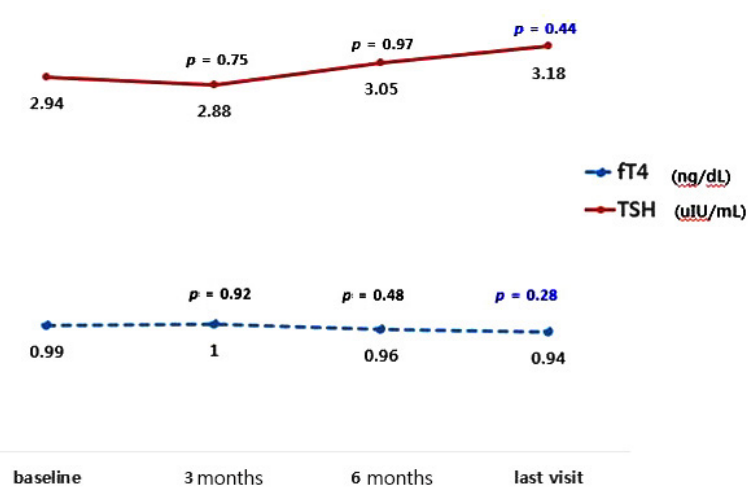

Figure 1. The longitudinal change of the mean fT4 (from $0.99 \pm 0.25$ to $0.94 \pm 0.71 \mathrm{ng} / \mathrm{dL}, p=0.28$ ) and TSH (from $2.94 \pm 1.32$ to $3.18 \pm 1.21$ $\mu \mathrm{lU} / \mathrm{mL}, p=0.44)$ levels in children with epilepsy on ketogenic diet showed no significant change from baseline to the last follow-up test during the mean 1.9-year follow-up period. fT4, free thyroxine; TSH, thyroid-stimulation hormone. 
Table 2. Difference of the fT4 and TSH levels from baseline to the last follow-up according to the potential variables

\begin{tabular}{|c|c|c|c|c|c|}
\hline Potential variables & $\mathrm{N}$ & fT4-D & $p$-value & TSH-D & $p$-value \\
\hline \multicolumn{6}{|l|}{ Sex } \\
\hline Male & 17 & $-0.07 \pm 0.21$ & \multirow[t]{2}{*}{0.93} & $0.55 \pm 1.39$ & \multirow[t]{2}{*}{0.24} \\
\hline Female & 11 & $0.02 \pm 0.09$ & & $-0.23 \pm 1.19$ & \\
\hline \multicolumn{6}{|l|}{ Age of $\mathrm{Sz}$ onset } \\
\hline$<1$ year & 16 & $-0.11 \pm 0.21$ & \multirow[t]{2}{*}{$0.02^{*}$} & $0.72 \pm 1.48$ & \multirow{2}{*}{$0.04^{*}$} \\
\hline$\geq 1$ year & 12 & $0.03 \pm 0.07$ & & $-0.39 \pm 0.83$ & \\
\hline \multicolumn{6}{|l|}{ Age of AEDs start } \\
\hline$<1$ year & 13 & $-0.09 \pm 0.23$ & \multirow[t]{2}{*}{0.05} & $0.77 \pm 1.45$ & \multirow[t]{2}{*}{0.05} \\
\hline$\geq 1$ year & 15 & $0.02 \pm 0.08$ & & $-0.30 \pm 1.01$ & \\
\hline \multicolumn{6}{|l|}{ Age of KD start } \\
\hline$<2$ years & 12 & $-0.18 \pm 0.18$ & \multirow[t]{2}{*}{$<0.01^{*}$} & $1.33 \pm 1.07$ & \multirow[t]{2}{*}{$<0.01^{*}$} \\
\hline$\geq 2$ years & 16 & $0.04 \pm 0.09$ & & $-0.57 \pm 0.88$ & \\
\hline \multicolumn{6}{|l|}{ Duration of $\mathrm{Sz}$} \\
\hline$<3$ years & 14 & $-0.09 \pm 0.16$ & \multirow[t]{2}{*}{0.14} & $0.44 \pm 1.58$ & \multirow[t]{2}{*}{0.64} \\
\hline$\geq 3$ years & 14 & $-0.01 \pm 0.18$ & & $0.51 \pm 1.09$ & \\
\hline \multicolumn{6}{|l|}{ Type of AEDs } \\
\hline CBZ, OXC, VPA, PB & 15 & $-0.04 \pm 0.23$ & \multirow[t]{2}{*}{0.19} & $-0.04 \pm 1.09$ & \multirow[t]{2}{*}{0.27} \\
\hline Others & 13 & $-0.07 \pm 0.09$ & & $0.57 \pm 1.58$ & \\
\hline \multicolumn{6}{|l|}{ Types of seizures } \\
\hline Single & 16 & $0.01 \pm 0.14$ & \multirow[t]{2}{*}{0.08} & $-0.17 \pm 1.20$ & \multirow[t]{2}{*}{0.06} \\
\hline Multiple & 12 & $-0.13 \pm 0.19$ & & $0.79 \pm 1.38$ & \\
\hline \multicolumn{6}{|l|}{ Frequency of Sz } \\
\hline$\leq 50 \%$ per week & 9 & $-0.06 \pm 0.21$ & 0.92 & $0.97 \pm 1.13$ & 0.05 \\
\hline Nearly daily & 19 & $-0.05 \pm 0.16$ & & $-0.10 \pm 1.33$ & \\
\hline MRI lesion & & & & & \\
\hline No & 8 & $-0.08 \pm 0.19$ & 0.64 & $-0.02 \pm 0.82$ & 0.71 \\
\hline Yes & 20 & $-0.04 \pm 0.17$ & & $0.35 \pm 1.52$ & \\
\hline Ratio of $K D$ & & & & & \\
\hline$\leq 3: 1$ & 15 & $-0.08 \pm 0.21$ & 0.93 & $0.29 \pm 1.24$ & 0.72 \\
\hline $4: 1$ & 13 & $-0.01 \pm 0.11$ & & $0.19 \pm 1.51$ & \\
\hline Urine ketone & & & & & \\
\hline$\leq 3+$ & 12 & $-0.07 \pm 0.17$ & 0.80 & $0.32 \pm 1.39$ & 0.69 \\
\hline $4+$ & 16 & $-0.04 \pm 0.19$ & & $0.18 \pm 1.36$ & \\
\hline Reduction of Sz & & & & & \\
\hline$\geq 50 \%$ & 17 & $-0.08 \pm 0.17$ & 0.23 & $0.23 \pm 1.12$ & 0.58 \\
\hline$<50 \%$ & 11 & $0.01 \pm 0.17$ & & $0.27 \pm 1.71$ & \\
\hline Duration of $K D$ & & & & & \\
\hline$<2$ year & 14 & $-0.05 \pm 0.18$ & 0.70 & $0.23 \pm 1.45$ & 0.84 \\
\hline$\geq 2$ years & 14 & $-0.05 \pm 0.18$ & & $0.26 \pm 1.28$ & \\
\hline $\mathrm{BMI}$ & & & & & \\
\hline$<85 \%$ & 21 & $-0.08 \pm 0.19$ & 0.10 & $0.23 \pm 1.39$ & 0.88 \\
\hline$\geq 85 \%$ & 7 & $0.03 \pm 0.05$ & & $0.29 \pm 1.29$ & \\
\hline Total cholesterol & & & & & \\
\hline$\leq 200 \mathrm{mg} / \mathrm{dL}$ & 13 & $0.18 \pm 0.08$ & $0.04^{*}$ & $-0.60 \pm 0.83$ & $<0.01^{*}$ \\
\hline$>200 \mathrm{mg} / \mathrm{dL}$ & 15 & $-0.11 \pm 0.22$ & & $0.98 \pm 1.29$ & \\
\hline Triglyceride & & & & & \\
\hline$\leq 300 \mathrm{mg} / \mathrm{dL}$ & 14 & $0.04 \pm 0.11$ & $<0.01^{*}$ & $-0.63 \pm 0.72$ & $<0.01^{*}$ \\
\hline$>300 \mathrm{mg} / \mathrm{dL}$ & 14 & $-0.14 \pm 0.18$ & & $1.12 \pm 1.27$ & \\
\hline
\end{tabular}

Values are presented as mean \pm standard deviation unless otherwise indicated.

fT4-D, difference of free thyroxine; TSH, thyroid-stimulation hormone; TSH-D, difference of thyroid-stimulation hormone; Sz, seizure; AEDS, antiepileptic drugs; KD, ketogenic diet; CBZ, carbamazepine; OXC, oxcarbazepine; VPA, valproate; PB, phenobarbital; MRI, magnetic resonance imaging; BMl, body mass index.

${ }^{*} p<0.05$.

\section{Discussion}

We discovered that the serum levels of fT4 $(-0.05 \pm 0.18 \mathrm{ng} / \mathrm{dL}, p$ $=0.28)$ and TSH $(0.24 \pm 1.35 \mu \mathrm{lU} / \mathrm{mL}, p=0.44)$ exhibited no sig- nificant longitudinal change in children with medically intractable epilepsy during KD therapy in our tertiary hospital-based population of pediatric epilepsy (mean duration, $1.9 \pm 1.5$ years). However, a younger age of seizure onset $(<1$ year of age; T4, $-0.11 \pm 0.21$ 
$\mathrm{ng} / \mathrm{dL}, p=0.02 ; \mathrm{TSH}, 0.72 \pm 1.48 \mu \mathrm{lU} / \mathrm{mL}, p=0.04)$, earlier initiation of KD therapy ( $<2$ years of age; $\mathrm{fT} 4,-0.18 \pm 0.18 \mathrm{ng} / \mathrm{dL}, p<$ $0.01 ; \mathrm{TSH}, 1.33 \pm 1.07 \mu \mathrm{IU} / \mathrm{mL}, p<0.01)$, and higher levels of serum total cholesterol $(>200 \mathrm{mg} / \mathrm{dL} ; \mathrm{fT} 4,-0.11 \pm 0.22 \mathrm{ng} / \mathrm{dL}, p=$ $0.04 ; \mathrm{TSH}, 0.98 \pm 1.29 \mu \mathrm{lU} / \mathrm{mL}, p<0.01)$ and triglyceride $(>300$ $\mathrm{mg} / \mathrm{dL} ; \mathrm{fT} 4,-0.14 \pm 0.18 \mathrm{ng} / \mathrm{dL}, p<0.01 ; \mathrm{TSH}, 1.12 \pm 1.27 \mu \mathrm{lU} / \mathrm{mL}$, $p<0.01$ ) were associated with a longitudinally significant decrease in thyroid function (decreased fT4 and increased TSH levels) from baseline to last follow-up during the KD.

There was just one study about the thyroid function of children (mean age, $7.3 \pm 4.3$ years) receiving KD for at least one year until now. ${ }^{12}$ This study reported that hypothyroidism was diagnosed in $16.7 \%(20 / 120)$ of patients during KD therapy, and that baseline TSH elevation and female sex were independent risk factors for development of hypothyroidism in epileptic children. These results were different from our results; baseline TSH elevation was found in $11.7 \%$ of these patients, which could influence the change of thyroid hormone. Our study can be considered the first longitudinal cohort study of thyroid function in pediatric epilepsy on KD from start to last visit in Korea.

The majority of the studies about the effects of antiepileptic drugs on thyroid function have been performed in adult patients. Different thyroid dysfunctions and subclinical hypothyroidism have been reported in adults. ${ }^{5}$ However, there are limited and conflicting data about the influence of AEDs on thyroid function in children. ${ }^{7,8}$ Valproate, carbamazepine, and phenobarbital are effective and widely used AEDs for the treatment of many types of epilepsy in children. ${ }^{9}$ The effect of valproate on serum thyroid hormone levels has been controversial. Both significantly decreased fT4 levels and significantly increased TSH levels were previously reported in patients during valproate therapy. ${ }^{6,8}$ On the contrary, other studies have demonstrated that both fT4 and TSH levels were unaffected in patients treated with valproate. ${ }^{13,14}$ Incidence of subclinical hypothyroidism was reported at a rate of $25.2 \%$ in patients treated with valproate with a duration of treatment between 6 and 24 months. ${ }^{15}$ The changes induced by long term administration of valproate appear to be transient and reversible. Carbamazepine typically causes reduced levels of fT4, but has variable effects on levels of TSH. ${ }^{6,8,14}$ The changes induced by carbamazepine appear to return to normal values after the withdrawal of medication. ${ }^{7,8}$ Subclinical hypothyroidism was previously reported in $8.2 \%$ of 61 children receiving carbamazepine. ${ }^{6}$ Oxcarbazepine, a 10-keto analog of carbamazepine, was developed to attempt to reduce the side effects that have been observed with administration of carbamazepine. Several studies have demonstrated that serum fT4 levels were significantly reduced in patients receiving oxcarbazepine, whereas TSH concentrations remained unchanged in children. ${ }^{7,8}$ The effect of oxcarbazepine on thyroid function may be short term, transient, and less significant than the effect of carbamazepine. ${ }^{8,14}$ Compared to control groups, children with epilepsy receiving chronic treatment with phenobarbital were found to have significantly reduced fT4 levels but unchanged TSH concentrations. ${ }^{6,7}$ However, the changes in TSH concentrations were less marked than those in patients treated with valproate. ${ }^{13} \mathrm{~A}$ previous study has shown that the increased TSH concentrations returned to normal values after the withdrawal of phenobarbital. ${ }^{7}$ Thus, the effects of phenobarbital on thyroid function appear reversible. Data on the effects of levetiracetam on thyroid function are very limited in children. ${ }^{13}$ On the whole, the effects on thyroid function induced by valproate and carbamazepine appear to be more marked than those induced by phenobarbital and oxcarbazepine. ${ }^{13}$

AEDs affect thyroid hormone levels through several mechanisms. A decrease in serum concentrations of thyroid hormones may be due to induction of the hepatic P450 enzyme system, resulting in accelerated clearance of thyroid hormones. ${ }^{16}$ Others interfere with the hypothalamic-pituitary-thyroid axis, ${ }^{17}$ and positive feedback mechanism of the axis may not be activated by this decrease in fT4 and free triiodothyronine levels. Thus, TSH levels in these patients remain normal. Another effect of carbamazepine on thyroid hormones may be due to interference with the competitive binding of thyroid hormone to thyroxine-binding globulin. ${ }^{8}$ Increase in TSH with valproate treatment might be due to $g$-aminobutyric acid (GABA) -stimulating properties of valproate. GABA inhibits release of somatostatin, which inhibits TSH secretion. ${ }^{7}$ Valproate might also lead to zinc and selenium deficiencies which are important enzymes in thyroid hormone synthesis, but the evidence is insufficient. ${ }^{7}$ Additional thyroidal pathways other than hypothalamic-pituitary axis alterations might also be involved in thyroid hormone metabolism. It is known that the KD-induced enhancement of energy metabolism compensates for a metabolic deficiency that compromises GABAergic inhibition. ${ }^{18} \mathrm{KD}$ may re-establish energy stores important for GABAergic output, and may help prevent rundown of post-synaptic $G A B A_{A}$ receptors. Therefore, we can presume that the KD-induced GABAergic augmentation and possible insufficiency of micronutrients may affect the thyroid hormone metabolism or synthesis. Our study also suggests that children with a younger age of seizure onset, earlier start of KD, and hyper- 
lipidemia (cholesterol or triglyceride) may be vulnerable to the negative effect of thyroid function during KD therapy.

Thyroid hormone abnormalities associated with AEDs result in subclinical hypothyroidism, which is defined as a mild elevation of TSH levels ( $5-25 \mu \mathrm{lU} / \mathrm{mL})$ in the presence of normal thyroid hormone levels, ${ }^{6}$ and appears to be benign with no effect on neuropsychological functions. ${ }^{19}$ Subclinical hypothyroidism was previously reported in $8.2 \%$ of 61 children receiving carbamazepine. ${ }^{6}$ Although the need for treatment of subclinical hypothyroidism remains controversial, replacement therapy is not justified in children with mild elevation of TSH levels, no goiter, and negative antithyroid antibodies. ${ }^{19}$ Although the children on KD treatment in this study had lower thyroid hormone levels during the follow-up period, these changes were all within normal reference ranges. In addition, their TSH levels did not change, and none of these hormonal changes led to any clinical symptoms in the follow-up period of $1.9 \pm 1.5$ years. Therefore, the clinical significance of low serum thyroid hormone levels is unclear. More long-term studies are needed to detect any subtle changes in asymptomatic individuals.

Our study had some limitations. First, we did not study the effect of discontinuation of KD on thyroid function. Second, we were not able to test a control group due to ethical reasons. However, our study was performed longitudinally using baseline and the last follow-up values to lessen the effect of this limitation. Lastly, although our patients had no change of their AEDs during KD, we consider that the effect of AEDs on thyroid function was not completely excluded.

In conclusion, overall thyroid function was not significantly decreased during KD therapy from baseline to the last visit in children with medically intractable epilepsy. However, children with a younger age of seizure onset, earlier initiation of KD, and higher levels of cholesterol and triglyceride exhibited a significant decrease in $\mathrm{fT} 4$ levels and increase in TSH levels during KD therapy. Augmented concern and careful monitoring of the serum levels of fT4/TSH should be recommended in children with these risk factors.

\section{Acknowledgements}

This study was supported by a 2017 research grant from Pusan National University Yangsan Hospital.

\section{References}

1. Hsieh LP, Huang CY. Trends in the use of antiepileptic drugs in
Taiwan from 2003 to 2007: a population-based national health insurance study. Epilepsy Res 2011;96:81-8.

2. Thvilum $M$, Brandt $F$, Brix $T H$, Hegedüs $L$. A review of the evidence for and against increased mortality in hypothyroidism. Nat Rev Endocinol 2012;8:417-24.

3. Aygün F, Ekici B, Aydinli N, Aydin BK, Baş F, Tatli B. Thyroid hormones in children on antiepileptic therapy. Int I Neurosci 2012;122:69-73.

4. Surks Ml, Sievert R. Drugs and thyroid function. N Eng/ / Med 1995;333:1688-94.

5. Kafadar İ, Kılıç BA, Arapoglu M, Yalçın K, Dalgıç N. Evaluation of thyroid hormones in children receiving carbamazepine or valproate: a prospective study. I Child Neurol 2015;30:63-8.

6. Eirís-Puñal J, Del Río-Garma M, Del Río-Garma MC, Lojo-Rocamonde S, Novo-Rodríguez I, Castro-Gago M. Long-term treatment of children with epilepsy with valproate or carbamazepine may cause subclinical hypothyroidism. Epilepsia 1999;40:1761-6.

7. Castro-Gago M, Novo-Rodríguez MI, Gómez-Lado C, Rodríguez-García J, Rodríguez-Segade $S$, Eiris-Puñal J. Evolution of subclinical hypothyroidism in children treated with antiepileptic drugs. Pediatr Neurol 2007;37:426-30.

8. Vainionpää LK, Mikkonen K, Rättyä J, et al. Thyroid function in girls with epilepsy with carbamazepine, oxcarbazepine, or valproate monotherapy and after withdrawal of medication. Epilepsia 2004;45:197-203.

9. de Silva M, MacArdle B, McGowan M, et al. Randomised comparative monotherapy trial of phenobarbitone, phenytoin, carbamazepine, or sodium valproate for newly diagnosed childhood epilepsy. Lancet 1996;347:709-13.

10. Freeman JM, Kossoff EH. Ketosis and the ketogenic diet, 2010: advances in treating epilepsy and other disorders. Adv Pediatr 2010;57:315-29.

11. Felton EA, Cervenka MC. Dietary therapy is the best option for refractory nonsurgical epilepsy. Epilepsia 2015;56:1325-9.

12. Kose E, Guzel O, Demir K, Arslan N. Changes of thyroid hormonal status in patients receiving ketogenic diet due to intractable epilepsy. IJ Pediatr Endocrinol Metab 2017;30:411-6.

13. Yılmaz U, Y Ylmaz TS, Akınc G, Korkmaz HA, Tekgül H. The effect of antiepileptic drugs on thyroid function in children. Seizure 2014;23:29-35.

14. Isojärvi Jl, Turkka J, Pakarinen AJ, Kotila M, Rättyä J, Myllylä W. Thyroid function in men taking carbamazepine, oxcarbazepine, or valproate for epilepsy. Epilepsia 2001;42:930-4.

15. Mikati MA, Tarabay H, Khalil A, Rahi AC, El Banna D, Najjar S. Risk factors for development of subclinical hypothyroidism during valproic acid therapy. J Pediatr 2007;151:178-81.

16. Connell JM, Rapeport WG, Gordon S, Brodie MJ. Changes in circulating thyroid hormones during short-term hepatic enzyme induction with carbamazepine. Eur I Clin Pharmacol 1984;26:453-6.

17. Verrotti A, Scardapane A, Manco R, Chiarelli F. Antiepileptic drugs and thyroid function. J Pediatr Endocrinol Metab 2008;21:401-8.

18. Bough K. Energy metabolism as part of the anticonvulsant mechanism 
Yun-Jin Lee, et al. Thyroid Hormone in Children on a Ketogenic Diet 105

of the ketogenic diet. Epilepsia 2008;49 Suppl 8:91-3.

19. Monzani A, Prodam F, Rapa A, et al. Endocrine disorders in childhood and adolescence. Natural history of subclinical hypothyroidism in chil- dren and adolescents and potential effects of replacement therapy: a review. Eur J Endocrinol 2012;168:R1-11. 